Published in final edited form as:

Qual Life Res. 2018 November ; 27(11): 3021-3035. doi:10.1007/s11136-018-1941-1.

\title{
Evaluation of opioid use among patients with back disorders and arthritis
}

\author{
Corey J. Hayes ${ }^{1,2}$, Nalin Payakachat ${ }^{1}$, Chenghui Li ${ }^{1}$ \\ ${ }^{1}$ Division of Pharmaceutical Evaluation and Policy, Department of Pharmacy Practice, University \\ of Arkansas for Medical Sciences College of Pharmacy, 4301 W. Markham St., \# 522, Little Rock, \\ AR 72205, USA \\ ${ }^{2}$ Division of Health Services Research, Psychiatric Research Institute, University of Arkansas for \\ Medical Sciences College of Medicine, Little Rock, AR, USA
}

\section{Abstract}

Purpose-Long-term opioid use for chronic pain has increased, but limited evidence exists on its benefits. Evaluation of long-term benefits in pain is based on patient-reported measures such as health-related quality of life (HRQoL). This study examined the long-term effects of opioid use on HRQoL and its subdomains in patients with back pain or arthritis by comparing opioid users to non-opioid users for three metrics: (1) any opioid use, (2) duration of opioid use, and (3) average daily morphine equivalent dose.

\begin{abstract}
Methods-A nationally representative sample of cancer-free adults with chronic back pain or arthritis was selected. Using the 12-Item Short Form Survey, HRQoL measures of Mental Component Score (MCS), Physical Component Score (PCS), and individual subdomains were assessed at baseline and 1 year later. Opioid users were matched to non-opioid users in a 1:1 greedy match using propensity scores estimated based on many patient demographics and baseline HRQoL measures.
\end{abstract}

Results-At year one, PCS was significantly lower among opioid users, mostly driven by bodily pain subdomain; MCS was not different. Short-term opioid users ( $<1$ month) had higher MCS while long-term users ( $\geq 1$ month) had lower PCS. Low-dose $[<20$ morphine milligram equivalents (MME)/day] opioid use was associated with lower PCS, while no difference was found between high dose ( $\geq 20 \mathrm{MME} /$ day) and non-opioid users. However, most differences were not clinically significant.

\footnotetext{
Chenghui Li, cli@uams.edu.

Conflict of interest Dr. Hayes was supported by the Translational Training in Addiction [1T32 DA 022981]. Dr. Payakachat received a research grant from 7-Hybrid Cultivation, an honorarium for service as a paid consultant to Roche Ltd., service as a consultant to CBPartners, and ownership of stock in Pfizer. Drs. Hayes and Li declare that they have no conflict of interest.

Research involving human and animal participants This article utilizes public-use data from the Agency for Healthcare Research and Quality (AHRQ). Therefore, it does not contain any studies with human participants or animals performed by any of the authors.

Electronic supplementary material The online version of this article (https://doi.org/10.1007/s11136-018-1941-1) contains supplementary material, which is available to authorized users.
} 
Conclusions-Long-term opioid use is not associated with improvements in HRQoL. Clinicians should carefully evaluate the need for opioid use, especially long-term use in managing chronic back pain and arthritis.

\section{Keywords}

Opioids; Health-related quality of life; Osteoarthritis; Rheumatoid arthritis; Back pain

\section{Introduction}

Back pain and arthritis are common chronic pain conditions; the prevalence of each is on the rise [1]. The percentage of patients with back pain increased from 3.9 to $10.2 \%$ over the 14year period from 1992 to 2006 [2]. During 2010-2012, 52.5 million US adults reported clinician-diagnosed arthritis, and by 2040 , that number is expected to rise to 78.4 million [1]. Other than surgical interventions and disease modifying anti-rheumatic drugs, most treatments for arthritis and back pain do not alter the cause of the pain.

Opioid analgesics are common treatments for back pain and both common forms of arthritis, osteoarthritis (OA), and rheumatoid arthritis (RA) [3]. Prescription opioid use in patients with RA has hovered around 35\% since 2005; however, chronic opioid use has risen from $2 \%$ in 2005 to $12 \%$ in 2014 [4]. In 2003, 31\% of patients with OA received opioids while in 2009 , it rose to $40 \%$ [5]. Using national survey data, one study found that opioid use for back and neck pain increased from 19 to 29\% from 1999 to 2010 [6].

Limited evidence exists on whether or not opioids are effective long-term for back pain and arthritis, making the decision for clinicians to initiate opioids difficult in light of their potential abuse [7, 8]. Effectiveness can be measured by several different endpoints, most of which are self-reported including pain level, functioning, and health-related quality of life (HRQoL) $[9,10]$. Evidence of the effectiveness of long-term opioid use on HRQoL is particularly important as HRQoL gives insight into the patient's perspective on their pain limitations, and physical and mental functional limitations. Instruments measuring HRQoL are versatile in that they not only provide the overall physical and mental component scores for HRQoL but also allow for assessment of the subdomains of these component scores.

The lack of evidence on the effectiveness of long-term opioid use on HRQoL on a national scale results from the scarcity of such information. Fortunately, the Medical Expenditure Panel Survey (MEPS) is one of the rare nationally representative sources of information on long-term effectiveness (roughly a 1-year time span) of opioid exposure in regards to HRQoL. This study, therefore, aims to fill the evidence gap on the long-term effects of opioid use on HRQoL and its subdomains in patients with back pain, RA, and/or OA by comparing opioid users to non-opioid users for three metrics: (1) any opioid use, (2) duration of opioid use, and (3) average daily morphine equivalent dose. Specifically, we hypothesize that HRQoL will not differ between opioid users and non-opioid users. We also hypothesize that only short durations of opioid use will be associated with higher HRQoL and that higher average daily morphine equivalent dose will be associated with lower HRQoL. 


\section{Methods}

\section{Data sources}

This study used a retrospective cohort study design. Data were obtained from MEPS, a nationally representative survey of non-institutionalized civilians in the US. MEPS has two major components: the household component and the insurance component. In this study, various public-use data files from the household component were used.

The household component of MEPS collects demographic characteristics, income, employment, health conditions, health status, and use of medical services as well as a wide array of additional health information that is supplemented by the household members' medical providers upon approval of the respondent. A panel design is used, covering 2 calendar years over five rounds [11]. About 15,000 households are represented within each panel. In rounds 2 and 4 , a supplemental survey called the adult self-administered questionnaire ( $S A Q$ ), was given to all respondents 18 years or older and includes information on HRQoL.

For this study, the Longitudinal, Medical Conditions, and Prescribed Medicine Files from 2010 to 2014 were used. The Longitudinal Files house data were collected over the 2-year period for each participant in the panel. The Medical Conditions Files contains ICD-9 and Clinical Classification Codes (CCC) for the medical conditions reported by the household members including in which rounds of the panel that condition was reported. The Prescribed Medicines Files include information on prescription medications used by members of the household and details of the medications were confirmed with the individual's pharmacy upon his/her consent [12]. Information gathered about each prescription includes the medication name, quantity dispensed, days' supply, National Drug Code (NDC), and cost of the prescription. NDC is a three-segment number that uniquely identified a drug.

This study was approved by the Institutional Review Board at the University of Arkansas for Medical Sciences.

\section{Study sample}

A cohort of adult participants with either back pain $(C C C=205), \mathrm{RA}(\mathrm{CCC}=202)$, or OA $(\mathrm{CCC}=203)$ for at least three of the five rounds (to ensure chronicity of disease) were derived from the Medical Conditions Files [13]. Exclusion criteria included: (1) cancer diagnosis in any of the five rounds except for non-melanoma skin cancer $(\mathrm{CCC}=011-022$, 024-045), (2) not in-scope with data collection for all five rounds of the survey, (3) ineligible for the SAQ in both rounds 2 and 4 of the survey, (4) missing scores for MCS and PCS in rounds 2 and 4, (5) missing scores for each of the subdomains of the MCS and PCS scores, (6) missing NDC for any prescription medication, and (7) opioid use in round 1, used to ensure new opioid users.

\section{Opioid exposure}

Multum Lexicon Drug Classes and NDCs were used to determine opioid exposure. Multum Lexicon Drug Classes classify medications based on therapeutic drug classes while NDCs 
are precise medication identification numbers used to describe the drug, strength, and formulation [14]. In accordance with the Lemke study, Multum classes 60 ("Narcotic Analgesics") and 191 ("Narcotic Analgesic Combinations") were used for this study [15]. In addition, Multum classes 59 ("Miscellaneous Analgesics") and 63 ("Analgesic Combinations") may also contain opioids; NDCs were used to cross-reference with these Multum classes to obtain additional opioid exposure.

Total opioid days' supply and months of use were determined for each participant in rounds 2 through 4 , which represents a 12-month period. If months of opioid use exceeded 12, it was assumed that some opioid prescriptions overlapped and a cap of 12 months was applied. Around $28 \%$ of opioids prescriptions had a missing days' supply. To impute missing days' supply, we first derived the average quantity per day by type and dosage form for each opioid from prescriptions with non-missing days' supply. Because average quantity per day is likely to also depend on pain severity, the averages were obtained based on reported pain limitations in round 2. Answer options for pain limitations include: "Not At All," "A Little Bit," "Moderately," "Quite A Bit," or "Extremely." Similarly to the Stockbridge et al. study, these were collapsed into four categories: none, mild, moderate, and severe with answer options "Quite a Bit" and "Extremely" being classified as severe [16]. In cases where $<10$ prescriptions were available to derive the average quantity per day for a certain opioid type and dosage form, the average quantity per day was instead calculated from the Pharmetrics LifeLink Plus database [17]. To derive the imputed days' supply, the total quantity of the prescription was divided by the imputed quantity per day. Because Schedule II opioids cannot have a days' supply > 30, the imputed days' supply for Schedule II opioids was capped at 30 days. Similarly, the days' supply for non-Schedule II opioids was capped at 90 because they cannot be dispensed with a days' supply > 90 days [18].

Opioid users from rounds 2-4 were further classified by the number of months of use and average daily dose. For comparison, opioid users were classified as short-term users $(<1$ month) or long-term users ( $\geq 1$ month), determined by a recent analysis showing a sharp rise in long-term use at 1 month [19]. Opioid fills were converted to morphine milligram equivalents (MMEs). MMEs were then summed for all opioid prescriptions per participant and divided by the participant's total opioid days' supply to get average daily dose [20]. Opioid users were categorized as low-dose users ( $<20$ MME daily) and higher-dose users ( $\geq$ 20 MME daily), determined based on increased risk of overdose in patients taking over 20 MME/day [21].

\section{Outcome measures}

HRQoL-MEPS includes the SF-12v2, an instrument to measure HRQoL, which has recently been validated in patients with non-cancer pain [22]. The SF-12v2 contains two summary measures of HRQoL, the Mental Component Summary (MCS) and the Physical Component Summary (PCS), each ranging from 0 to 100 with higher scores representing better mental or physical health [23]. MEPS contains the overall PCS and MCS scores. PCS and MCS scores are derived from differential weightings of eight subdomains: role functioning (RF), physical functioning (PF), bodily pain (BP), and general overall health perceptions (GH), role emotional (RE), social functioning (SF), vitality (VT), and overall 
mental health (MH) [24]. PCS weights RF, PF, BP, and GH more heavily, while MCS weights RE, SF, VT, and MH more heavily. The subdomains scores were calculated using public SAS code and were converted to norm-based scores from the U.S. population mean (50) and standard deviation (10) [25]. Therefore, the subdomain scores also range from 0 to 100 where higher scores are indicative of improved health. Changes in both summary scores and the subdomain scores were analyzed.

Because statistical significance is not always indicative of clinical significance, minimal clinically important improvements (MCIIs), obtained from published literature in patients with RA, low back pain, or related conditions, were used to establish clinical significance. The MCII reported for PCS ranges from 3.3 to 7.2 for patients with RA and low back pain [26, 27]. The MCII for the MCS is 3.77 as derived from low back pain patients [27]. For subdomains, only MCIIs for PF and BP were found. The MCII for the PF subdomain is 7.111.6 as derived from RA and total knee replacement patients [26, 28]. The MCII for BP subdomain is 4.9 as derived from a RA population [26]. Any differences in PCS, MCS, and their respective subdomain scores greater than the MCIIs between comparison groups (e.g., opioid users vs. non-opioid users) were considered clinically significant differences. If a range (of MCIIs) is found, the lowest value was used. For subdomains without reported MCIIs, an MCII of 5 was used since it represents a change that is $50 \%$ of the norm-based standard deviation, which is considered a medium effect size [29]. Each of the questions that compose the subdomains are detailed in Table 1 [30].

\section{Covariates}

Covariates included demographics (age, race, gender, education level, and marital status), health insurance status, income level, geographic location, and baseline measures from round 2 of the outcome variables as defined previously. To account for any longitudinal changes in comorbidity burden that could lead to differential use of opioids after round 2 and outcomes in round 4, we also included the Deyo adaptation of the Charlson Comorbidity Index $(0,1, \geq 2)$ assessed over the entire 2-year period, [31] and pain-related medications exposure from rounds 2 through 4 (antidepressants [76, 208, 209, 250, 306, 307, 308], COX-2 inhibitors [278], non-steroidal anti-inflammatory drugs (NSAIDs) [61], skeletal muscle relaxants $[178,179]$, and anti-anxiety medications $[68,69,70])$. Chronic pain diagnoses (RA, OA, or back pain) were also included.

\section{Propensity score development/matching}

To balance the observed differences between opioid users and non-opioid users, propensity scores were estimated using logistic regression to predict opioid use based on the covariates listed above. Opioid users were 1:1 matched to non-opioid users based on propensity scores using a greedy match algorithm without replacement [32]. Imbalances in the variables included in the logistic regression to derive the propensity score were assessed using both $p$ values and standardized differences. The matching process was repeated and nonlinear terms of the covariates and interactions were added until all standardized differences were $<10 \%$ [33]. A C statistic $\geq 0.7$ was used to assess the overall goodness of fit of the logistic regression [34]. 


\section{Statistical analysis}

Demographic characteristics of the sample were first represented as unweighted percentages for each demographic variable. Survey commands in SAS (PROC SURVEYFREQ and PROC SURVEYMEANS) were used to incorporate survey weights (variables within MEPS) to generate nationally representative estimates. After the groups were successfully matched, PCS and MCS scores and their subdomain scores were analyzed using linear regressions, with the independent variables being (in separate regressions) any opioid use (use vs. no use), months of use ( $\geq 1$ month, $<1$ month vs. no use), or dose ( $\geq 20$ MME daily, $<20$ MME daily, vs. no use). Since participants are matched based on opioid use, final linear models comparing months of use and dose included adjustment for the quartiles of the propensity score. To adjust for multiple comparisons, $p$ values for subdomain comparisons were adjusted using Hochberg's method [35]. Survey command in SAS (PROC SURVEYREG) were used to estimate the least square means between the two groups while incorporating strata of paired participants from the propensity score matching. All analyses were conducted using SAS v9.3 (SAS Institute Inc., Cary, NC) at a two-sided significance level of 0.05 .

\section{Results}

Participant characteristics

1871 eligible participants were identified, with 690 opioid users and 1181 non-opioid users (Fig. 1). The characteristics of these participants are shown in Table 2. Of both opioid and non-opioid users, most were white, female, between the ages of 50 and 65, and married with private insurance. A higher percent of opioid users, compared to non-opioid users, have chronic back pain, use anti-anxiety medications, skeletal muscle relaxants, antidepressants, and non-steroidal anti-inflammatory medications. In round 2 of MEPS, a higher percent of opioid users, compared to non-opioid users, report lower scores for each of the eight subdomains of HRQoL.

\section{Propensity score matching}

The final logistic regression model used to create propensity scores for opioid use had 152 linear variables and interaction terms. The $\mathrm{C}$ statistic for this logistic regression was above the goal threshold level of 0.7 (0.787). During propensity score matching, 326 of the 690 (47.2\%) opioid users matched to a non-opioid user in the 1:1 greedy match. After matching, no statistically significant differences existed between the two groups in regards to the covariates with all standardized differences being $\leq 10 \%$ (eTable 1).

\section{HRQOL between opioid users and non-opioid users}

MCS and PCS-Among matched opioid users and non-opioid users, MCS in round 4 was not significantly different as shown in Fig. 2 (eTable 2: $\beta=0.622,95 \%$ CI -1.16 to $2.40, p$ $=.4931)$, but PCS in round 4 was significantly lower in opioid users compared to non-opioid users (Fig. 2) (eTable 2: $\beta=-2.26,95 \%$ CI -4.16 to $-0.37, p=.0194$ ).

Subdomains-None of the heavily weighted subdomains of the MCS in round 4 were significantly different as shown in Fig. 2 and eTable 2. However, the BP and RF subdomains 
were significantly lower among opioid users (eTable 2 : $\mathrm{BP}: \beta=-2.72,95 \% \mathrm{CI}-4.72$ to $0.72, p=.0077$; RF: $\beta=-1.89,95 \% \mathrm{CI}-3.71$ to $-0.08, p=.0411)$. PF and GH were not different between opioid users and non-opioid users. After adjustment of the $p$ values with the Hochberg's method, though, only BP remained significantly lower among opioid users compared to non-opioid users, as shown in Fig. 2 and eTable 2.

\section{HRQOL by duration of opioid use}

MCS and PCS-Among matched pairs of opioid users and non-opioid users, MCS and PCS were compared between non-opioid users $(N=326)$ and short-term opioid users $(<1$ month, $N=165$ ) and long-term opioid users ( $\geq 1$ month, $N=161$ ). MCS was significantly higher among short-term opioid users compared to non-opioid users, as shown in Fig. 3 (eTable 3: $\beta=2.29,95 \%$ CI $0.26-4.33, p=.0272$ ). MCS was not significantly different between long-term opioid users and non-opioid users (Fig. 3 and eTable 3). PCS was significantly lower among long-term opioid users compared to non-opioid users (Fig. 3) (eTable 3: $\beta=-5.77,95 \% \mathrm{CI}-7.92$ to $-3.61, p<.0001$ ); however, PCS was not significantly different (though trended towards improvement) between short-term opioid users and non-opioid users (Fig. 3 and eTable 3).

Subdomains-VT, RE, and MH scores are higher among short-term opioid users compared to non-opioid users (Fig. 3) (eTable 3: VT: $\beta=1.87,95 \%$ CI 0.08-3.67, $p$ $=.0411$; RE: $\beta=2.78,95 \%$ CI 0.63-4.92, $p=.0114 ; \mathrm{MH}: \beta=2.09,95 \%$ CI $0.15-4.03, p$ $=.0351)$, but SF was not significantly different between the two groups. After adjustment using the Hochberg's method, only the $p$ value for RE (adjusted $p=.0456$ ) remained significant when comparing short-term opioid users to non-opioid users. When comparing long-term opioid users to non-opioid users, VT and RE were not significantly different between the two. However, both SF and MH were significantly lower among long-term opioid users (SF: $\beta=-4.16,95 \% \mathrm{CI}-6.71$ to $-1.62, p=.0014$; $\mathrm{MH}: \beta=-2.53,95 \% \mathrm{CI}-$ 4.72 to $-0.35, p=.0233$ ). After adjustment using the Hochberg's method, only the $p$ value for SF (adjusted $p=.0028$ ) remained significant when comparing long-term opioid users to non-opioid users.

BP, PF, RF, and GH were not significantly different between short-term opioid users compared to non-opioid users (although each trended towards higher scores for each) (Fig. 3; eTable 3). However, each of these subdomains were significantly lower among long-term opioid users compared to non-opioid users (BP: $\beta=-7.10,95 \% \mathrm{CI}-9.36$ to $-4.85, p$ $<.0001$; PF: $\beta=-4.60,95 \% \mathrm{CI}-6.84$ to $-2.35, p<.0001$; RF: $\beta=-4.52,95 \% \mathrm{CI}-6.65$ to $-2.38, p<.0001$; GH: $\beta=-3.17,95 \% \mathrm{CI}-5.12$ to $-1.22, p=.0045)$. Even after adjustment with Hochberg's method, all the $p$ values for BP, PF, RF, and GH between longterm opioid users and non-opioid users remained significant.

\section{HRQOL by average daily dose in MMEs}

MCS and PCS-Among matched pairs of opioid users and non-opioid users, MCS and PCS were compared between non-opioid users $(N=326)$ and low-dose opioid users $(<20$ MMEs daily, $N=122$ ) and higher-dose opioid users ( $\geq 20$ MME daily, $N=204$ ). MCS was not significantly different between low-dose opioid users ( $<20$ MMEs daily) and higher- 
dose opioid users ( $220 \mathrm{MME}$ daily) compared to non-opioid users (Fig. 4 and eTable 4). PCS was significantly lower among low-dose opioid users compared to non-opioid users ( $\beta$ $=-2.84,95 \% \mathrm{CI}-5.19$ to $-0.49, p=.0178$ ). However, PCS was not significantly different between higher-dose opioid users and non-opioid users.

Subdomains-SF and MH were significantly lower among low-dose opioid users compared to non-opioid users (Fig. 4) (eTable 4: SF: $\beta=-2.96,95 \%$ CI -5.60 to $-0.32, p$ $=.0283 ; \mathrm{MH}: \beta=-2.39,95 \% \mathrm{CI}-4.68$ to $-0.09, p=.0417)$. However, both SF and MH were not significantly different between higher-dose opioid users and non-opioid users. Furthermore, none of the comparisons for average daily dose were significant for VT and RE. After correction with the Hochberg's method for multiple comparisons, SF, VT, RE, and MH were not significant.

$\mathrm{BP}, \mathrm{RF}$, and GH were significantly lower among low-dose opioid users compared to nonopioid users (Fig. 4) (eTable 4: BP: $\beta=-3.55,95 \%$ CI -6.05 to $-1.05, p=.0055$; RF: $\beta=$ $-3.09,95 \% \mathrm{CI}-5.34$ to $-0.84, p=.0072$; GH: $\beta=-2.31,95 \% \mathrm{CI}-4.46$ to $-0.16, p$ $=.0354)$. After correction of the $p$ values, only BP and RF scores remained significantly lower for low-dose opioid users compared to non-opioid users (BP: $p=.0055 ; \mathrm{RF}: p$ $=.0144)$. BH, RF, PF, and GH were not significantly different between higher-dose opioid users and non-opioid users.

\section{Discussion}

\section{HRQOL between opioid users and non-opioid users}

This study showed opioid users compared to non-opioid users do not significantly differ in their MCS scores, but PCS scores are negatively impacted by opioid use, primarily driven by worsened BP. It is worth noting, though, that neither of the results for PCS or BP reached a MCII (PCS: 3.3 and BP: 4.9); therefore, this negative statistically significant impact is not likely to be a clinically significant impact to the patient.

\section{HRQOL by duration of opioid use}

As to length of opioid use, this study showed that MCS was positively impacted by shortterm opioid use ( $<1$ month). This improvement is driven by the VT, RE, and MH subdomains (per unadjusted $p$ value). However, with long-term use ( $\geq 1$ month), MCS and each of its subdomains trend to be negatively impacted, with the SF subdomain becoming statistically lower compared to non-use. While PCS scores do not statistically differ between short-term opioid users and non-opioid users, long-term opioid users have statistically lower PCS, and each of the four heavily weighted subdomains for PCS (BP, RF, PF, and GH) were also significantly lower in round 4 compared to non-opioid users. This finding is supported by previous literature. A clinical review of 11 randomized controlled trials of opioid use for RA found that weak opioids may be effective for short-term use in RA, but the adverse effects may outweigh the benefits in long term use [36]. Furthermore, recent results from a 12-month randomized pragmatic trial evaluated opioid versus non-opioid medications in the treatment of chronic back, hip, or knee pain and found no differences in pain-related 
function, lower pain intensity among the non-opioid group, and higher medication-related adverse events with the opioid group [37].

\section{HRQOL by average daily dose in MMEs}

As to opioid dose, MCS and VT, SF, RE, and MH scores were not significantly impacted by differing doses. However, PCS and BP and RF subdomains are negatively impacted by lowdose use (<20 MME daily). Despite these statistically significant findings with PCS, BP, and RF, the findings do not reach clinical significance. PCS and the BP, RF, PF, and GH subdomains are not significantly different between those taking higher opioid doses ( $\geq 20$ MME) compared to non-opioid users. The interpretation of these dosing findings is unclear. One possible explanation is low dose of opioid use does not have enough therapeutic benefit to outweigh the extensive side-effect profile of opioids [45].

Overall, except for short-term use, opioid use does not seem to improve HRQoL long term. Previous studies have found similar results in various pain types. One study in patients with neuropathic pain found that metrics for health status and disability did not improve or were worse with opioid use [38]. Patients who used opioids for neuropathic pain did have higher PCS scores, although the difference did not reach a clinically significant threshold [39]. PCS, for our population, was lower among opioid users; however, as with the above study [38], the differences seen in our study are not considered a MCII for either the general population [40] or among those with certain pain conditions [41, 42] (MCII range 4-8). Another study found that opioid use was associated with higher disability in those with back pain as measured by the Roland-Morris Disability Questionnaire [43]. In regards to pain intensity, a recent study in Veterans with persistent pain found opioid prescriptions are associated with a lower likelihood of improvement in pain intensity [44].

\section{Confounding bias}

As an observational study, the risk of confounding is high. We have made concerted efforts to adjust for observed differences between the two groups. Propensity score methods were used, adjusting for covariates known to be associated with physical functionality, physical HRQoL, and opioid use such as comorbidity index, gender, and income level [15, 46, 47]. Since the outcome measures used in this study were evaluated twice within MEPS, (in rounds 2 and 4), outcome measures from round 2 were also used in the derivation of the propensity score to provide an equal baseline. To account for potential longitudinal changes in comorbidity burden, comorbidities reported over the entire 2-year period and exposure to several medication classes hypothesized to be associated with our outcome measures during rounds 2 through 4 were also used to derive the propensity scores. Nonetheless, residual confounding due to unmeasured variables may still exist. However, even if this omitted confounding bias leads to underestimation of some positive impact of opioid use, it does not seem to be substantial enough to overcome the negative impact for all opioid use measures, except short-term use.

\section{Limitations}

Our results should be interpreted in light of several limitations. First, reporting of prescription information is voluntary by the participant and not all patients' prescriptions 
records were confirmed with their pharmacies; hence, the true extent of opioid exposure may be underestimated. Second, analyses using propensity scores can only control for measured confounders that are included in the propensity score development and residual confounding could still exist due to unmeasured confounders. Third, the highest granularity for time of opioid use is the round, which limits our ability to determine the temporal relationship between opioid use and outcome in round 4. Fourth, the subdomains of the SF-12v2 are derived from fewer items than the SF-36, which may make the subdomains less reliable. However, the interpretation of the subdomains are intended to identify which are having the greatest influence on the overall MCS and PCS scores. Fifth, there were 1409 participants who were excluded due to either a missing values for MCS or PCS, its subdomains, or NDC at baseline or at followup. It is unclear the reason for the missing scores. It is also unclear the impact it might have on the results had these participants not had missing scores. In conducting chisquare differences on key demographic variables between the final sample and these 1409 that were excluded, age, gender, and geographic location were not statistically different between the two groups. However, race was statistically different (those excluded had roughly $4 \%$ higher number of African Americans). Sixth, in testing duration of opioid use and average daily dose, opioid users and non-opioid users were not rematched based on the cut points used in these two analyses. Instead, the final linear models adjusted for the quartiles of the propensity score. Lastly, due to sample size issues, heterogeneous treatment effects by pain diagnosis were not evaluated.

\section{Conclusions}

Overall, we did not find evidence that opioid use for back pain or arthritis improves HRQoL or its subdomains, except for a slight benefit with short-term use. With the benefits of prolonged opioid use still unclear and seeing the obvious risk associated with opioid use, prescribers should continually re-evaluate the individual benefit of continuing use in their patients.

\section{Supplementary Material}

Refer to Web version on PubMed Central for supplementary material.

\section{Funding}

Funding was provided by National Institute on Drug Abuse (Grant No. 1T32 DA 022981).

\section{References}

1. Hootman JM, Helmick CG, Barbour KE, Theis KA, \& Boring MA (2016). Updated projected prevalence of self-reported doctor-diagnosed arthritis and arthritis-attributable activity limitation among US adults, 2015-2040. Arthritis \& Rheumatology (Hoboken, N.J.), 68(7), 1582-1587. 10.1002/art.39692

2. Freburger JK, Holmes GM, Agans RP, Jackman AM, Darter JD, Wallace AS, et al. (2009). The rising prevalence of chronic low back pain. Archives of Internal Medicine, 169(3), 251-258. 10.1001/archinternmed.2008.543. [PubMed: 19204216]

3. Landsman-Blumberg PB, Katz N, Gajria K, D'Souza AO, Chaudhari SL, Yeung PP, et al. (2017). Health care resource use and cost differences by opioid therapy type among chronic noncancer pain patients. Journal of Pain Research, 10, 1713-1722. 10.2147/JPR.S130913. [PubMed: 28769587] 
4. Zamora-Legoff JA, Achenbach SJ, Crowson CS, Krause ML, Davis JM, \& Matteson EL (2016). Opioid use in patients with rheumatoid arthritis 2005-2014: A population-based comparative study. Clinical Rheumatology, 35(5), 1137-1144. 10.1007/s10067-016-3239-4. [PubMed: 27022929]

5. Wright EA, Katz JN, Abrams S, Solomon DH, \& Losina E (2014). Trends in prescription of opioids from 2003 to 2009 in persons with knee osteoarthritis. Arthritis Care \& Research, 66(10), 14891495. 10.1002/acr.22360. [PubMed: 24782079]

6. Mafi JN, McCarthy EP, Davis RB, \& Landon BE (2013). Worsening trends in the management and treatment of back pain. JAMA Internal Medicine, 173(17), 1573 10.1001/jamainternmed.2013.8992. [PubMed: 23896698]

7. Chaparro LE, Furlan AD, Deshpande A, Mailis-Gagnon A, Atlas S, \& Turk DC (2014). Opioids compared with placebo or other treatments for chronic low back pain. Spine, 39(7), 556-563. 10.1097/BRS.0000000000000249. [PubMed: 24480962]

8. Chou R, Rick Deyo F, Beth Devine M, Ryan Hansen R, Sullivan S, Jarvik JG, et al. (2014). The effectiveness and risks of long-term opioid treatment of chronic pain. Rockville, MD: Agency for Healthcare Research and Quality (US).

9. Breivik H, Borchgrevink PC, Allen SM, Rosseland LA, Romundstad L, Breivik Hals EK, et al. (2008). Assessment of pain. British Journal of Anaesthesia, 101(1), 17-24. 10.1093/bja/aen103. [PubMed: 18487245]

10. Katz N (2002). The impact of pain management on quality of life. Journal of Pain and Symptom Management, 24(1), S38-S47. 10.1016/S0885-3924(02)00411-6. [PubMed: 12204486]

11. Agency for Healthcare Research and Quality. (n.d.). Medical Expenditure Panel Survey background. Retrieved August 10, 2016, from https://meps.ahrq.gov/mepsweb/about_meps/ survey_back.jsp.

12. Agency for Healthcare Research and Quality. (n.d.). Medical Expenditure Panel Survey home. Retrieved May 3, 2016, from http://meps.ahrq.gov/mepsweb/.

13. Medical Expenditure Panel Survey Public Use File Search Results. (n.d.). Retrieved January 11, 2017, from https://meps.ahrq.gov/mepsweb/data_stats/download_data_filesresults.jsp? cboDataYear=All\&cboDataType $=1 \% 2 \mathrm{CHousehold}+$ Full + Year + File\&button YearandDataType $=$ Search \&cboPufNumber=All\&SearchTitle=Medical+Conditions

14. Multum Source Information. (n.d.). Retrieved from https://www.nlm.nih.gov/research/umls/ sourcereleasedocs/current/MMSL/.

15. Lemke M (2015). Prescription opioid use in the U.S. in 2012: Characterizing sustained vs. infrequent use using the medical expenditure panel survey. Retrieved from http:// www.lexjansen.com/sesug/2015/182_Final_PDF.pdf.

16. Stockbridge EL, Suzuki S, \& Pagán JA (2015). Chronic pain and health care spending: An analysis of longitudinal data from the Medical Expenditure Panel Survey. Health Services Research, 50(3), 847-870. 10.1111/1475-6773.12263. [PubMed: 25424348]

17. IMS Health. (n.d.). IMS LifeLink Health Plan Claims Database: Overview and study design issues. Retrieved November 21, 2015, from http://www.uams.edu/TRI/hsrcore/ Lifelink_Health_Plan_Claims_Data_DesignIssues_wcost_April2010\%5B1\%5D.pdf.

18. U.S Department of Justice Drug Enforcement Administration Office of Diversion Control. (2014). Rules-2014-Final rule: Rescheduling of hydrocodone combination products from schedule III to schedule II. Retrieved August 11, 2016, from https://www.deadiversion.usdoj.gov/fed_regs/ rules/2014/fr0822.htm.

19. Shah A, Hayes CJ, \& Martin BC (2017). Characteristics of initial prescription episodes and likelihood of long-term opioid use - United States, 2006-2015. MMWR Morbidity and Mortality Weekly Report, 66(10), 265-269. 10.15585/mmwr.mm6610a1. [PubMed: 28301454]

20. Hayes CJ, Hudson TJ, Phillips MM, Bursac Z, Williams JS, Austin MA, et al. (2015). The influence of propoxyphene withdrawal on opioid use in veterans. Pharmacoepidemiology and Drug Safety, 24(11), 1180-1188. 10.1002/pds.3851. [PubMed: 26248742]

21. Dowell D, Haegerich TM, \& Chou R (2016). CDC guideline for prescribing opioids for chronic pain - United States, 2016. MMWR. Recommendations and Reports, 65(1), 1-49. 10.15585/ mmwr.rr6501e1er. 
22. Hayes C, Bhandari N, Kathe N, \& Payakachat N (2017). Reliability and Validity of the medical outcomes study short form-12 version $2(\mathrm{SF}-12 \mathrm{v} 2)$ in adults with non-cancer pain. Healthcare, 5(2), 22 10.3390/healthcare5020022.

23. Ware J (2005). How to score version 2 of the SF-12 health survey (with a supplement documenting version 1). Lincoln, RI: Quality-Metric Inc.

24. Cheak-Zamora NC, Wyrwich KW, \& McBride TD (2009). Reliability and validity of the SF-12v2 in the medical expenditure panel survey. Quality of Life Research, 18(6), 727-735. 10.1007/ s11136-009-9483-1. [PubMed: 19424821]

25. Weblet Importer. (n.d.). Retrieved November 15, 2017, from http://gim.med.ucla.edu/FacultyPages/ Hays/utils/sf12v2-1.sas.

26. Ward MM, Guthrie LC, \& Alba MI (2014). Clinically important changes in short form 36 health survey scales for use in rheumatoid arthritis clinical trials: The impact of low responsiveness. Arthritis Care \& Research, 66(12), 1783-1789. 10.1002/acr.22392. [PubMed: 24980417]

27. Díaz-Arribas MJ, Fernández-Serrano M, Royuela A, Kovacs FM, Gallego-Izquierdo T, RamosSánchez M, et al. (2017). Minimal clinically important difference in quality of life for patients with low back pain. SPINE. 10.1097/BRS.0000000000002298.

28. Escobar A, Quintana JM, Bilbao A, Aróstegui I, Lafuente I, \& Vidaurreta I (2007). Responsiveness and clinically important differences for the WOMAC and SF-36 after total knee replacement. Osteoarthritis and Cartilage, 15(3), 273-280. 10.1016/j.joca.2006.09.001. [PubMed: 17052924]

29. Cohen J (1977). Statistical power analysis for the behavioral sciences. New York: Academic Press.

30. SF-12v2TM Health Survey. (n.d.). Retrieved from http://www.cscc.unc.edu/codi/forms/ UNLICOMMSFHSF12ShortFormHealthSurvey03192010.pdf.

31. Deyo RA, Cherkin DC, \& Ciol MA (1992). Adapting a clinical comorbidity index for use with ICD-9-CM administrative databases. Journal of Clinical Epidemiology, 45(6), 613-619. [PubMed: 1607900]

32. Austin PC (2008). Goodness-of-fit diagnostics for the propensity score model when estimating treatment effects using covariate adjustment with the propensity score. Pharmacoepidemiology and Drug Safety, 17(12), 1202-1217. 10.1002/pds.1673. [PubMed: 18972454]

33. Austin PC (2011). An introduction to propensity score methods for reducing the effects of confounding in observational studies. Multivariate Behavioral Research, 46(3), 399-424. 10.1080/00273171.2011.568786. [PubMed: 21818162]

34. LaValley MP (2008). Logistic regression. Circulation, 117(18), 2395-2399. 10.1161/ CIRCULATIONAHA.106.682658. [PubMed: 18458181]

35. Hochberg Y, \& Benjamini Y (1990). More powerful procedures for multiple significance testing. Statistics in Medicine, 9(7), 811-818. 10.1002/sim.4780090710. [PubMed: 2218183]

36. Whittle SL, Richards BL, \& Buchbinder R (2013). Opioid analgesics for rheumatoid arthritis pain. JAMA, 309(5), 485 10.1001/jama.2012.193412. [PubMed: 23385275]

37. Krebs EE, Gravely A, Nugent S, Jensen AC, DeRonne B, Goldsmith ES, et al. (2018). Effect of opioid vs nonopioid medications on pain-related function in patients with chronic back pain or hip or knee osteoarthritis pain. JAMA, 319(9), 872 10.1001/jama.2018.0899. [PubMed: 29509867]

38. Sites BD, Beach ML, \& Davis MA (2014). Increases in the use of prescription opioid analgesics and the lack of improvement in disability metrics among users. Regional Anesthesia and Pain Medicine, 39(1), 6-12. 10.1097/AAP.0000000000000022. [PubMed: 24310049]

39. Bostick GP, Toth C, Carr ECJ, Stitt LW, Morley-Forster P, Clark AJ, et al. (2015). Physical functioning and opioid use in patients with neuropathic pain. Pain Medicine (Malden, Mass.), 16(7), 1361-1368. 10.1111/pme.12702.

40. Warkentin LM, Majumdar SR, Johnson JA, Agborsangaya CB, Rueda-Clausen CF, Sharma AM, et al. (2014). Weight loss required by the severely obese to achieve clinically important differences in health-related quality of life: Two-year prospective cohort study. BMC Medicine, 12, 175 10.1186/ s12916-014-0175-5. [PubMed: 25315502]

41. Parker SL, Adogwa O, Mendenhall SK, Shau DN, Anderson WN, Cheng JS, et al. (2012). Determination of minimum clinically important difference (MCID) in pain, disability, and quality of life after revision fusion for symptomatic pseudoarthrosis. The Spine Journal, 12(12), 1122 1128. 10.1016/j.spinee.2012.10.006. [PubMed: 23158968] 
42. Parker SL, Mendenhall SK, Shau D, Adogwa O, Cheng JS, Anderson WN, et al. (2012). Determination of minimum clinically important difference in pain, disability, and quality of life after extension of fusion for adjacent-segment disease. Journal of Neurosurgery: Spine, 16(1), 6167. 10.3171/2011.8.SPINE1194. [PubMed: 21962034]

43. Ashworth J, Green DJ, Dunn KM, \& Jordan KP (2013). Opioid use among low back pain patients in primary care: Is opioid prescription associated with disability at 6-month follow-up? Pain, 154(7), 1038-1044. 10.1016/j.pain.2013.03.011. [PubMed: 23688575]

44. Dobscha SK, Lovejoy TI, Morasco BJ, Kovas AE, Peters DM, Hart K, et al. (2016). Predictors of improvements in pain intensity in a national cohort of older veterans with chronic pain. The Journal of Pain, 17(7), 824-835. 10.1016/j.jpain.2016.03.006. [PubMed: 27058162]

45. Benyamin R, Trescot AM, Datta S, Buenaventura R, Adlaka R, Sehgal N, et al. (2008). Opioid complications and side effects. Pain Physician, 11(2 Suppl), S105-S120. [PubMed: 18443635]

46. Bartoli A, Michna E, He E, \& Wen W (2015). Pain intensity and interference with functioning and well-being in subgroups of patients with chronic pain treated with once-daily hydrocodone tablets. Journal of Opioid Management, 11(6), 519-533. 10.5055/jom.2015.0305. [PubMed: 26728649]

47. Kea B, Fu R, Lowe RA, \& Sun BC (2016). Interpreting the national hospital ambulatory medical care survey: United States Emergency Department Opioid Prescribing, 2006-2010. Academic Emergency Medicine, 23(2), 159-165. 10.1111/acem.12862 [PubMed: 26802501] 


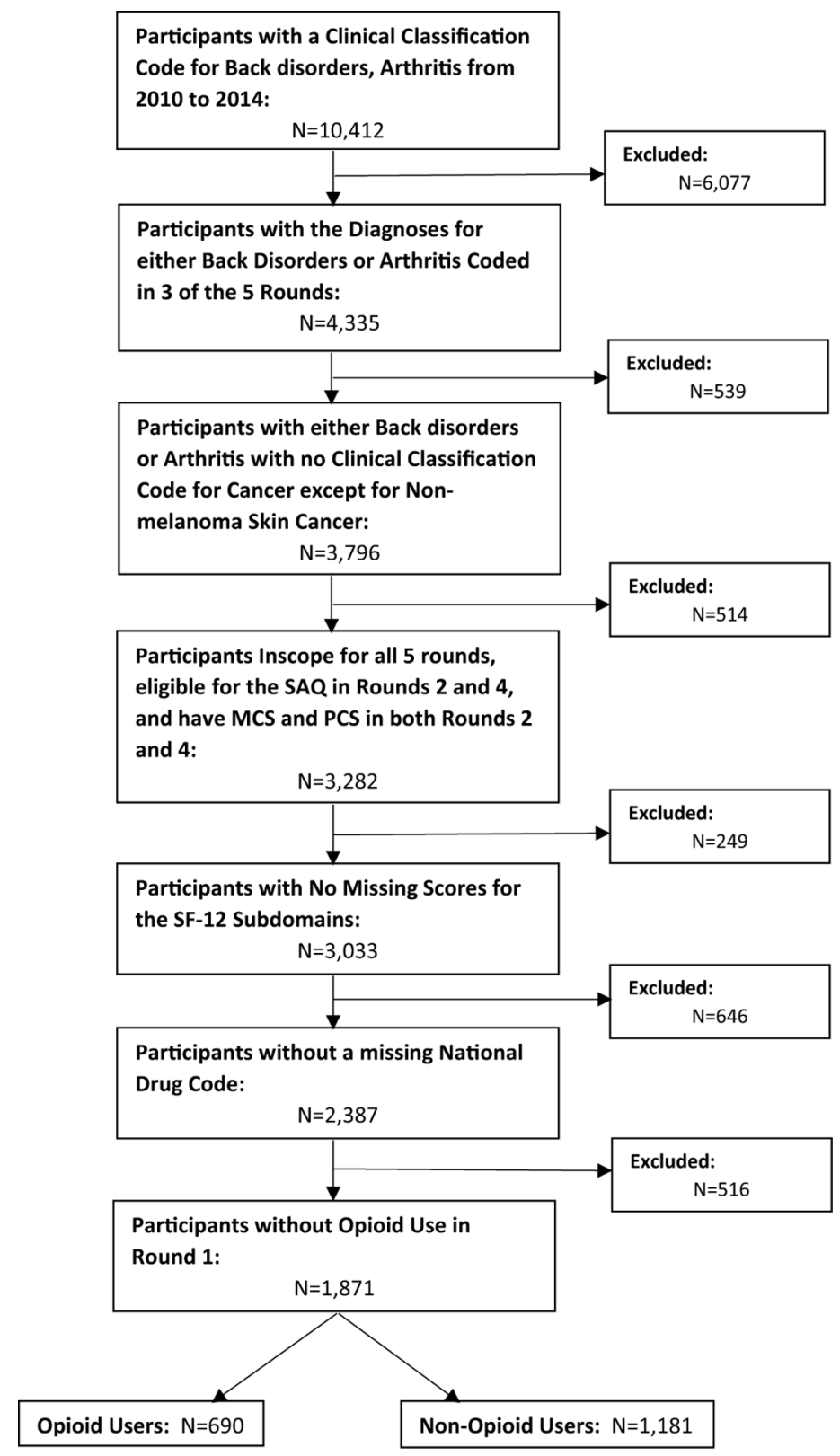

Fig. 1.

Patient flowchart with application of inclusion and exclusion criteria 


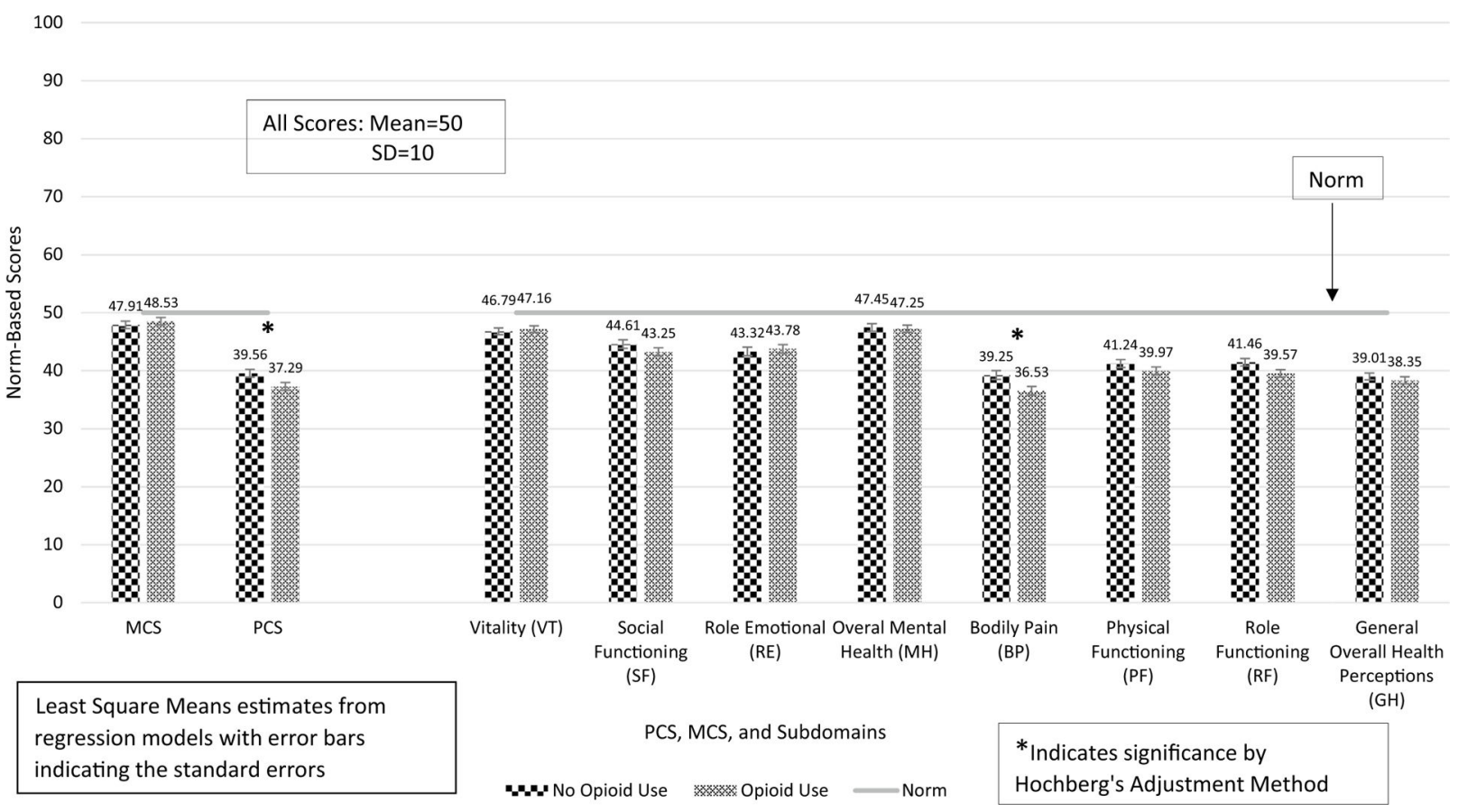

Fig. 2.

PCS, MCS, and their subdomain scores among matched pairs of opioid users with nonopioid users with arthritis and/or back pain 


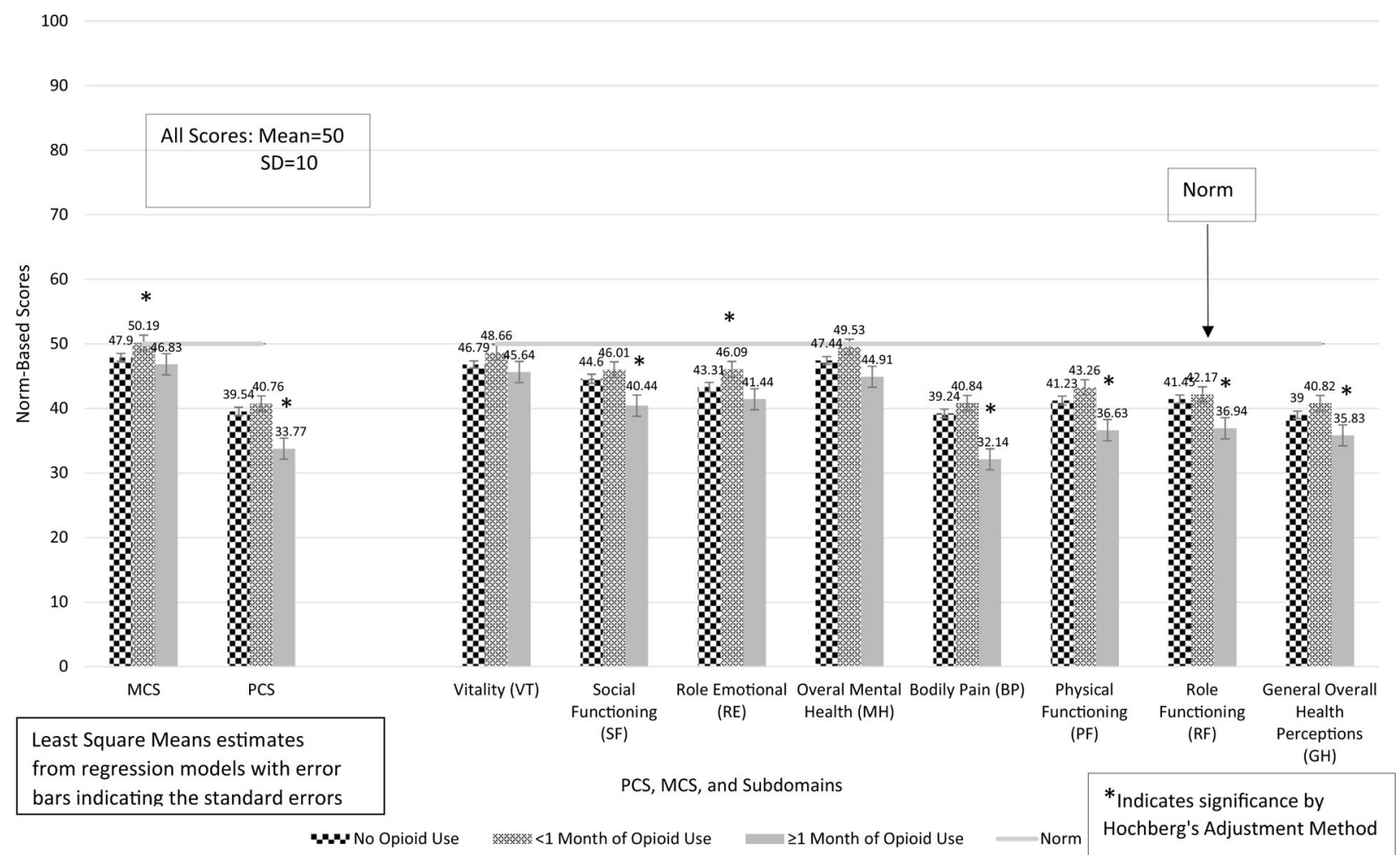

Fig. 3.

PCS, MCS, and their subdomain scores by length of opioid use among matched pairs of opioid users and non-opioid users with arthritis and back pain 


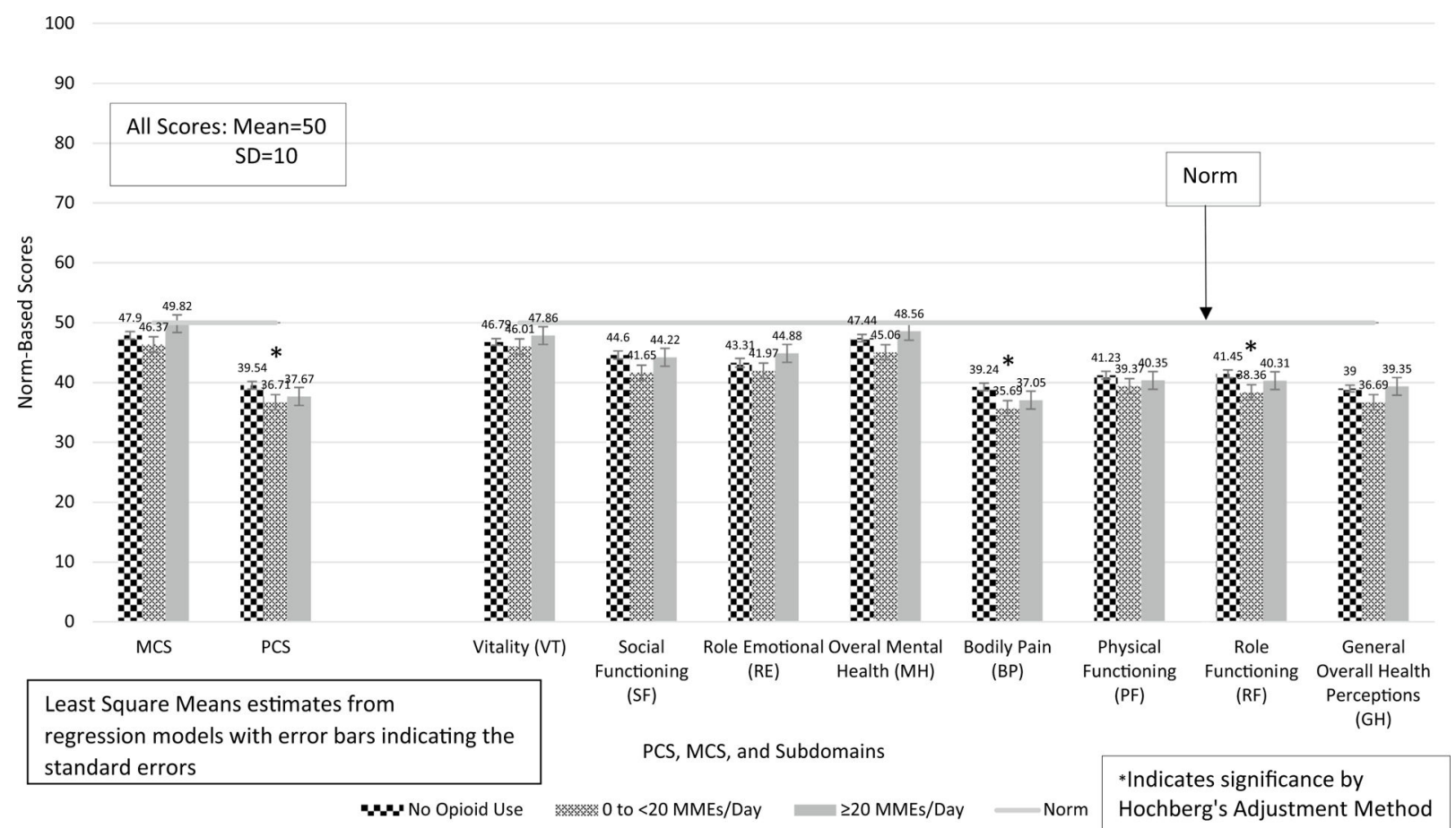

Fig. 4.

PCS, MCS, and their subdomain scores by dose of opioid use among matched pairs of opioid users and non-opioid users with arthritis and back pain 


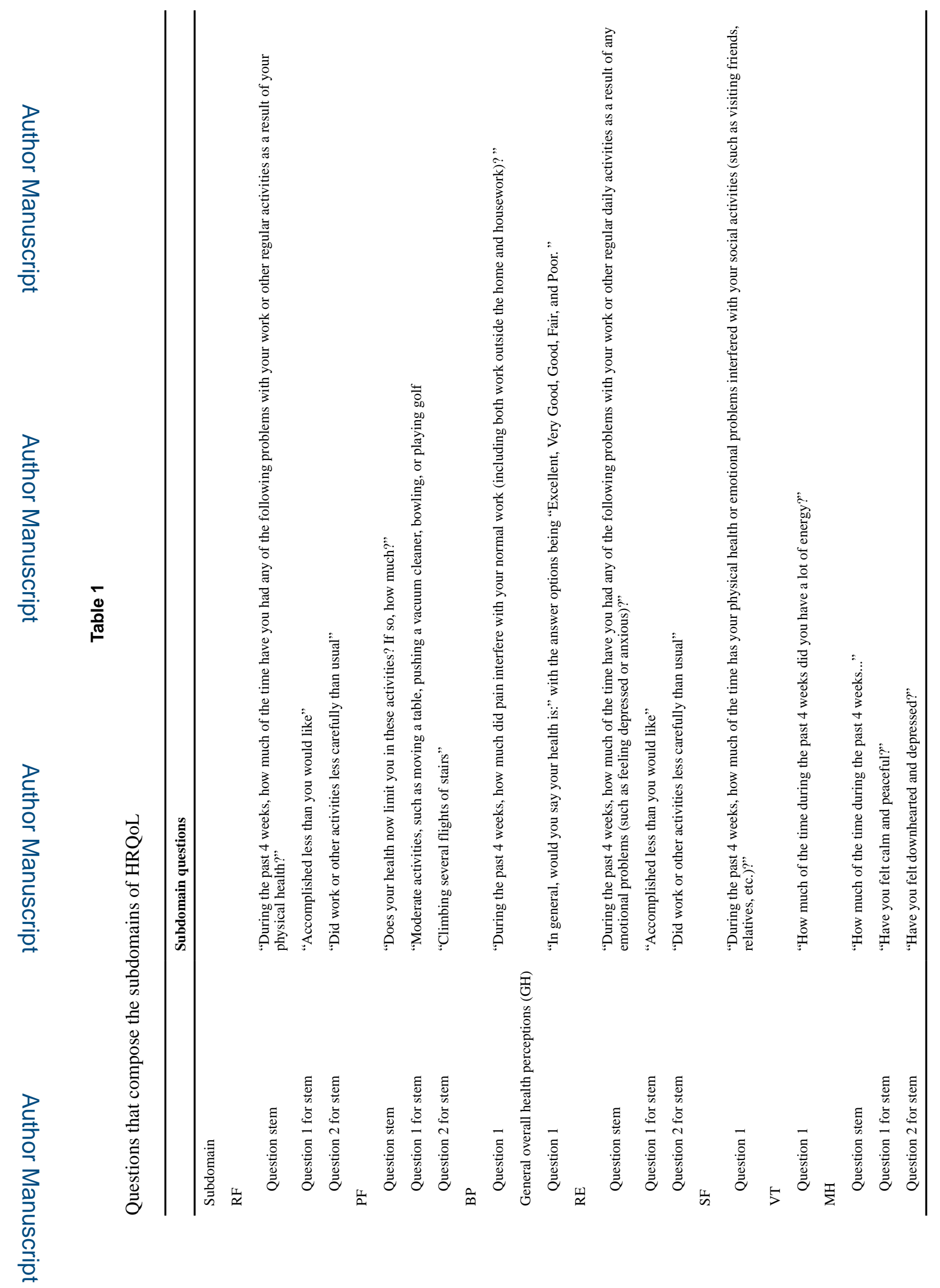

Qual Life Res. Author manuscript; available in PMC 2020 May 01. 


\section{을 \\ }

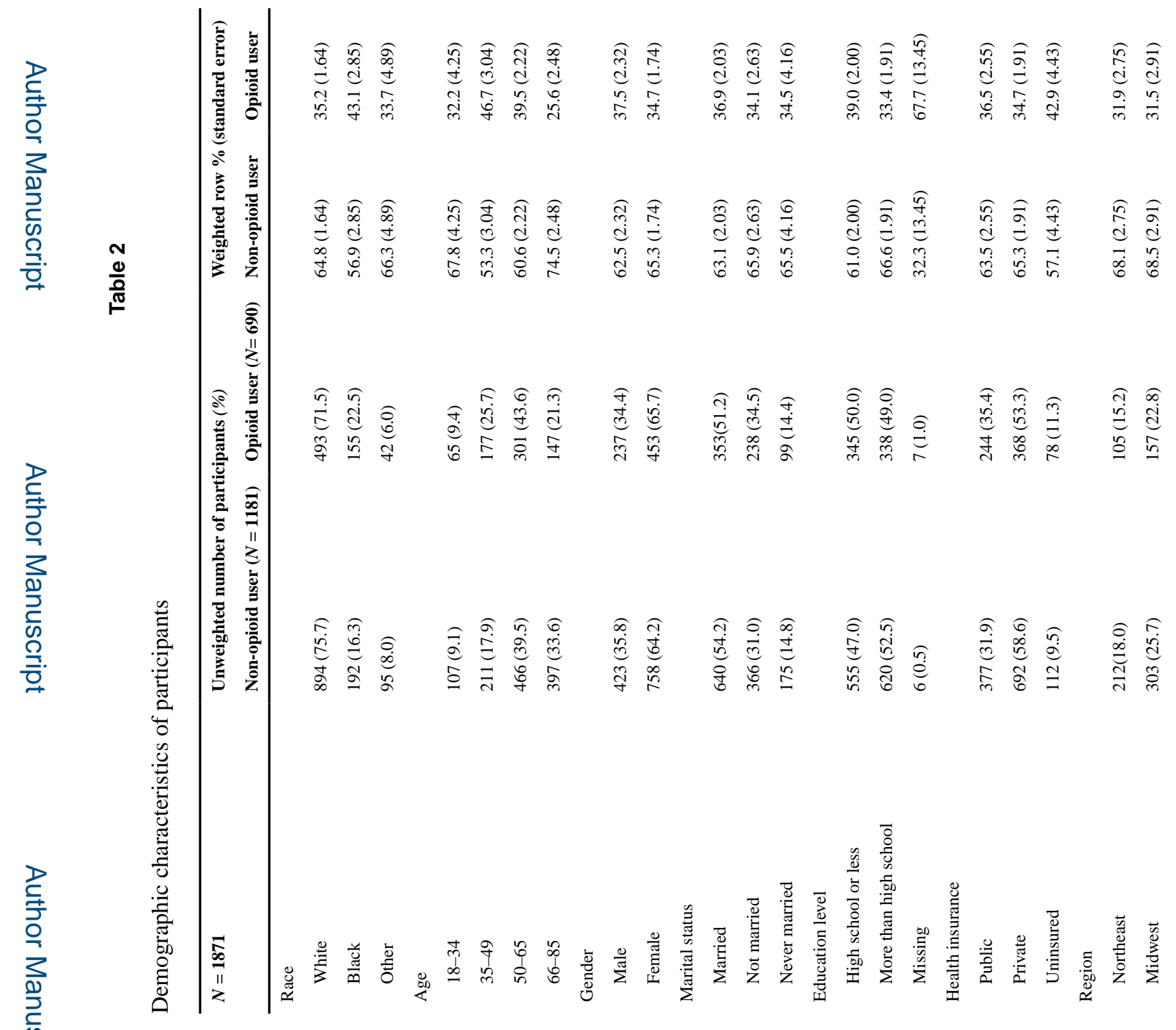

Qual Life Res. Author manuscript; available in PMC 2020 May 01. 
Hayes et al.

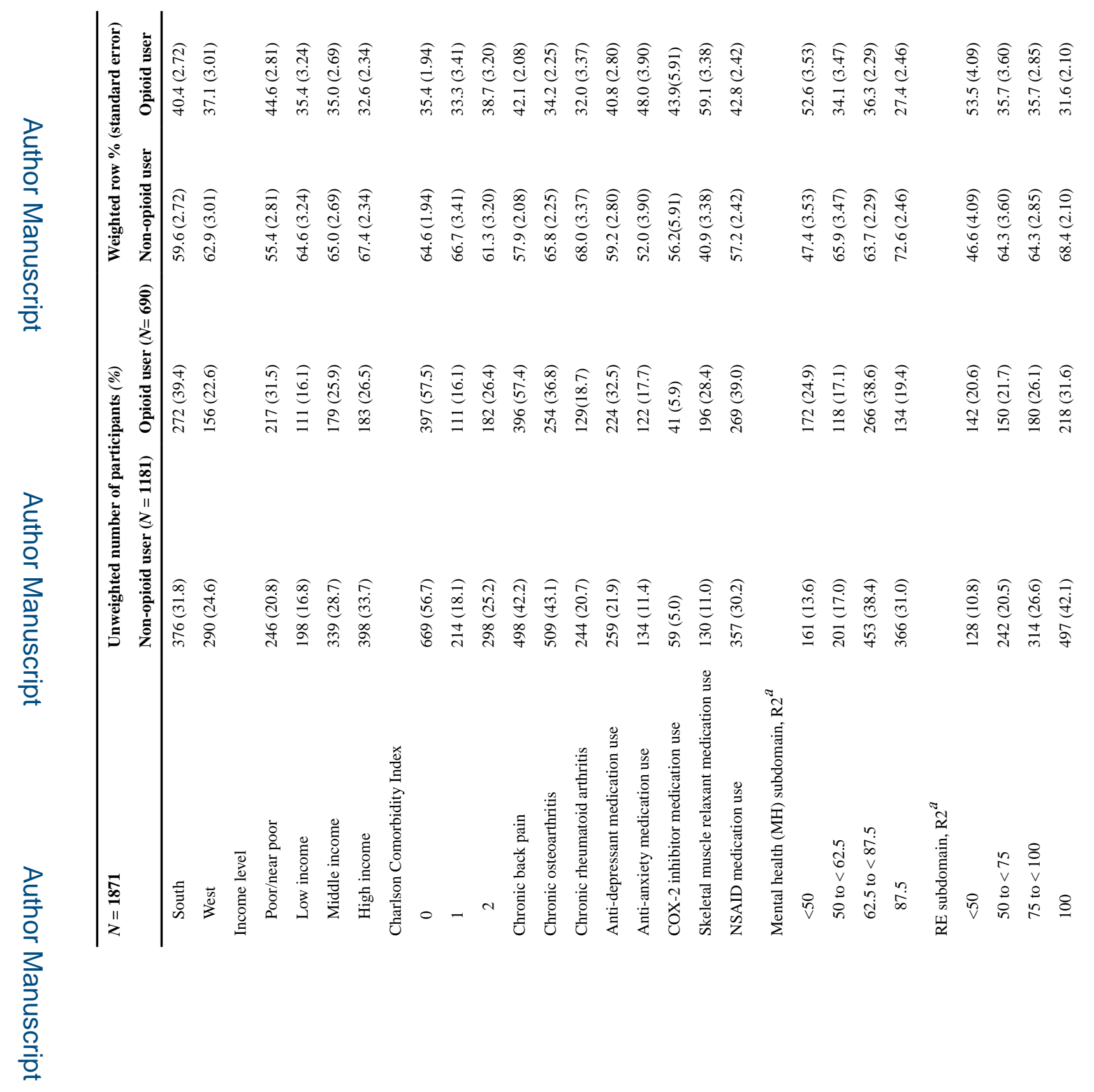

를

Qual Life Res. Author manuscript; available in PMC 2020 May 01. 


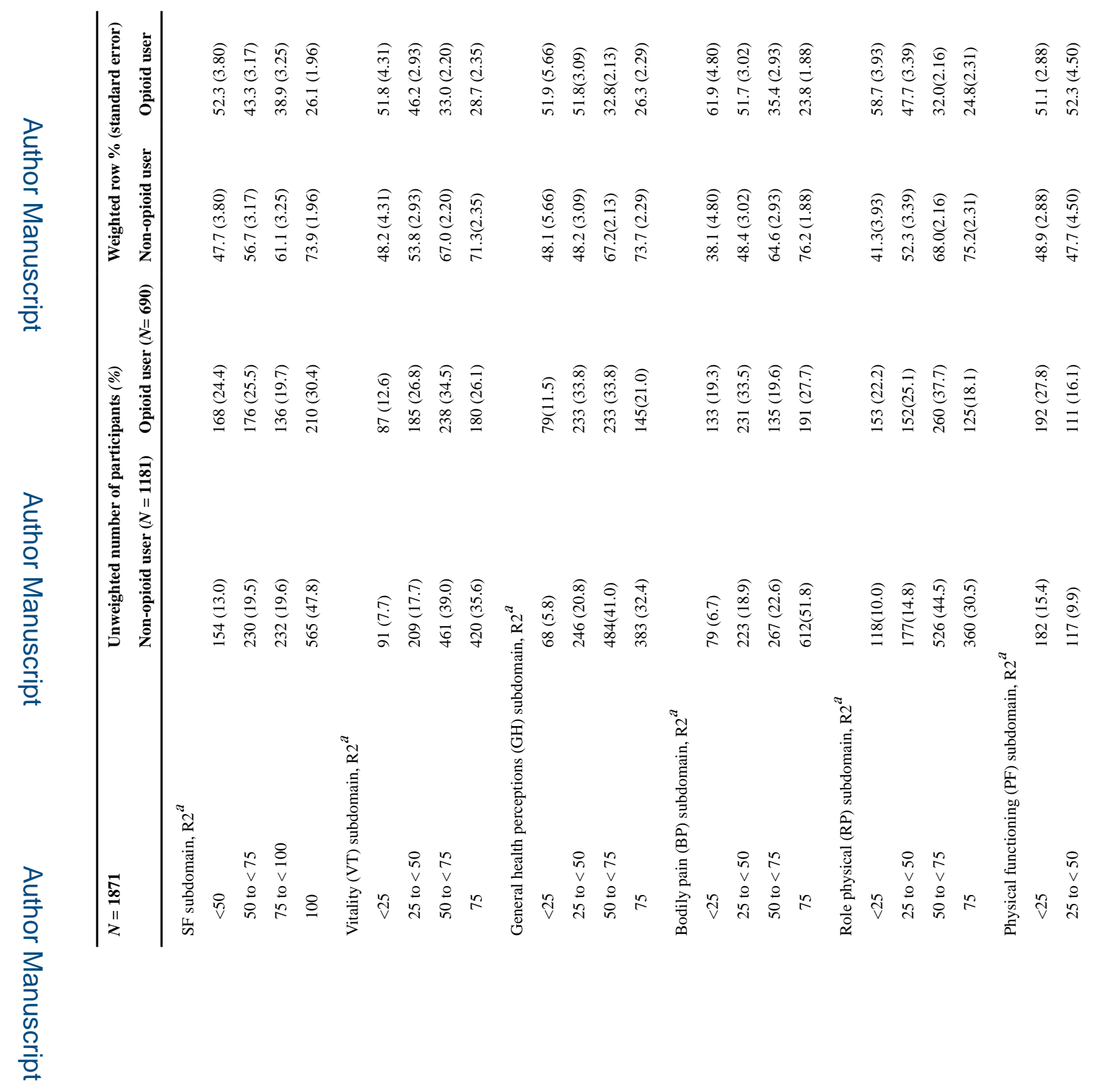

로을

Qual Life Res. Author manuscript; available in PMC 2020 May 01. 
Hayes et al.

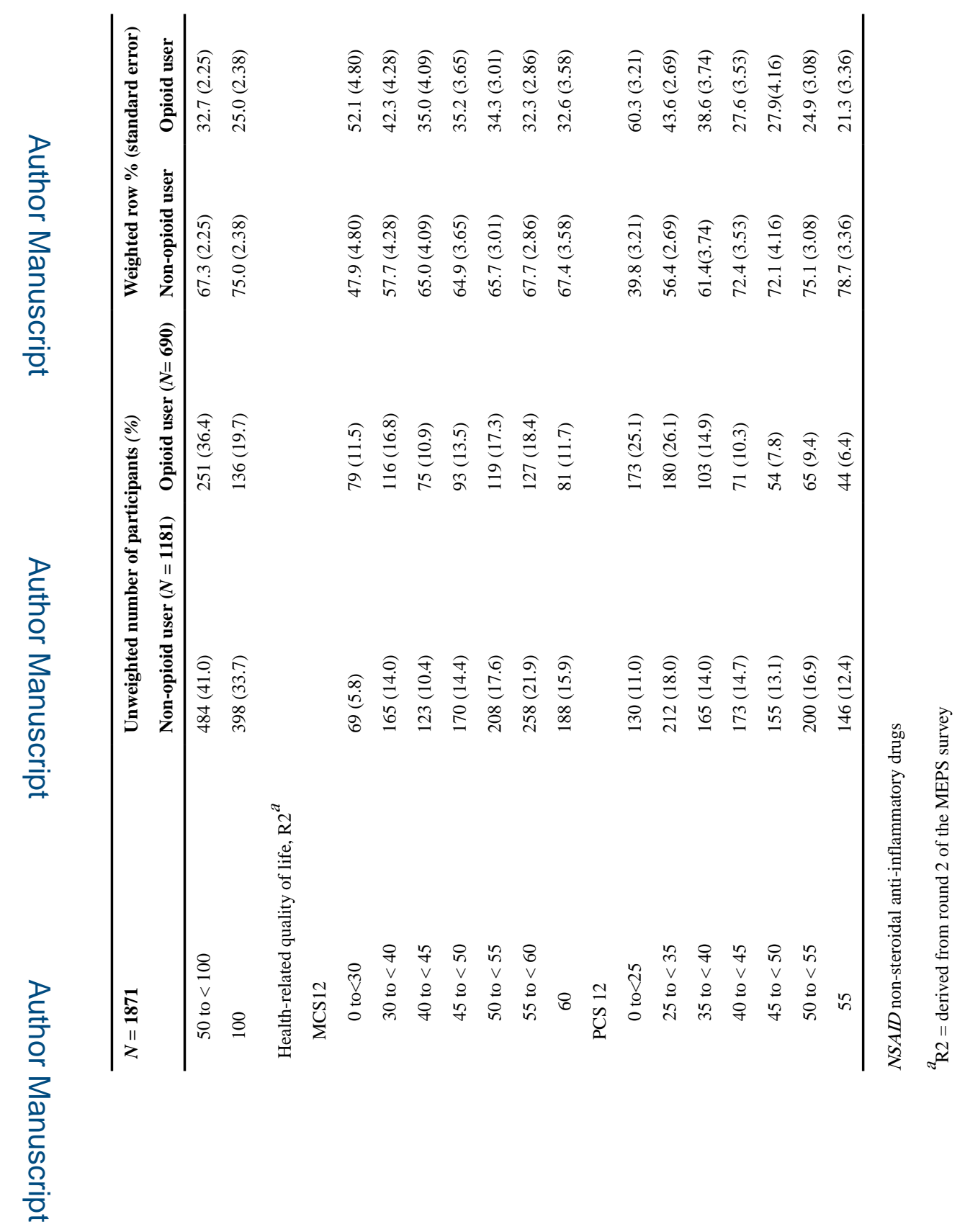

로을

Qual Life Res. Author manuscript; available in PMC 2020 May 01. 